\title{
WIENER'S BLACK BOX IN THE DYNAMIC IDENTIFICATION OF METALLURGICAL PROCESS WHICH IS BEING AUTOMATED
}

\section{Zhanbay Shuynshalyevych Uzdenbayev ${ }^{1}$, Aliya Ongarbayeva ${ }^{2}$, Aigul Yelepbergenova ${ }^{3}$}

\begin{abstract}
The use of physical and chemical models in metallurgic automatic control algorithms is rather complex. The reasons include a high rank of the multiple correlation, which requires sufficient aprioristic information; and the static character of most coefficients and variables. The result is a complex mathematical apparatus, and a development timeline that becomes so long that the project may not be useful. Schematically, the same processes may have different technological conditions and this lowers the unification ratio of solutions. Rather than focusing on the definition of the observed variable dependencies on each perturbation, the suggested method in this article involves the study of the behavior of the output variable, conditionally accepting that the system is affected by some common parameter (factor) that is the resultant action of perturbations. In keeping with this approach, we plot a series of ramps, form a system of mathematical models, calculate automatic regulator parameters, and create an adaptive control algorithm. An example of the method and results is presented in the article. Based on the adequacy of models using the initial data, this study found that the greatest value of divergence from the results of five experiments was $0.18 \%$.
\end{abstract}

UDC Classification: 681.5 DOI: http://dx.doi.org/10.12955/cbup.v4.857

Keywords: identification, ramp, mathematical model, adaptive regulator.

\section{Introduction}

The use of physical and chemical models in metallurgic automatic control algorithms is rather complex. The reasons include a high rank of the multiple correlation, which also requires sufficient aprioristic information; and the static character of most coefficients and variables. Such ways of modelling have been investigated in length, for example by Burovoi and Eliashberg (1964) and other researchers.

If one considers the process of roasting lead agglomerate, for example, the mathematical model of the process, taking into account the temperature in the agglomeration zone and the degree of desulfurization, can be shown as follows (Equation 1):

$$
\left.\begin{array}{l}
\frac{d C_{S}}{d t}=\frac{1}{T_{1}\left(Q_{s h}\right)}\left(C_{S}^{s h}-C_{S}\right)-F\left(\Theta, C_{S}, C_{O_{2}}\right) ; \\
\frac{d C_{O_{2}}}{d t}=\frac{1}{T_{1}\left(Q_{D}\right)}\left(\beta C_{O_{2}}^{D}-C_{O_{2}}\right)-a_{1} \frac{G}{V_{G}} F\left(\Theta, C_{S}, C_{O_{2}}\right) ; \\
\frac{d C_{S O_{2}}}{d t}=-\frac{1}{T_{2}\left(Q_{D}\right)} C_{S O_{2}}+a_{2} \frac{G}{V_{G}} F\left(\Theta, C_{S}, C_{O_{2}}\right) ; \\
\frac{d \Theta}{d t}=\frac{K_{y}\left(Q_{s h}, Q_{D}\right)}{T_{3}\left(Q_{s h}, Q_{D}\right)} G^{\prime}-\frac{1}{T_{3}\left(Q_{s h}, Q_{D}\right)} \Theta ; \\
F\left(\Theta, C_{S}, C_{O_{2}}\right)=k^{\prime}(\Theta) C_{S} C_{O_{2}}=k_{0}^{\prime} \exp \left(-\frac{E^{\prime}}{R T}\right) C_{S} C_{O_{2}} ; \\
T_{1}=\frac{G}{Q_{\mathrm{sh}}} ; \quad T_{2}=\frac{V_{G}}{\beta Q_{D}} ; \quad T_{3}=\frac{c^{p} G}{\sum_{i=1}^{2} Q_{i} C_{i}+k_{T P}} ; \quad K_{y}=\frac{q}{c^{p} G} T_{3} ;
\end{array}\right\}
$$

\footnotetext{
${ }^{1}$ Zhanbay Shuynshalyevych Uzdenbayev, Zhansugurov Zhetisu State University, Taldykorgan, Republic of Kazakhstan, jorajorin@mail.ru

${ }^{2}$ Aliya Ongarbayeva, Zhansugurov Zhetisu State University, Taldykorgan, Republic of Kazakhstan, aliaod 80@mail.ru

${ }^{3}$ Aigul Yelepbergenova, Zhansugurov Zhetisu State University, Taldykorgan, Republic of Kazakhstan, aigul_eu@mail.ru
} 
Where $C_{S}, C_{O 2}, C_{S_{O} 2}$ are sulfur, oxygen, and sulfur dioxide, respectively, and $\Theta$, the roasting zone temperature, $T_{1}, T_{2}, T_{3}$ - equivalent time constants (hours); $Q_{s h}$ - the flow of furnace-charge (tonnes per hour); $C_{S}^{s h}$ - sulphur concentration in furnace-charge (relative portion); $Q_{D}$ - air blasting flow (cubic meters per hour); $\beta$ - a dimensionless factor considering the thermal dilatation of gases; $C_{O 2}^{D}{ }^{-}$ the oxygen content in air blasting (tonnes per cubic meter); $\mathrm{a}_{1}, \mathrm{a}_{2}$ - stoichiometric factors; $G$ - mass of an agglomerate layer (tonnes); $V_{G}$ - fumes volume (cubic meters); $K_{y}$ - an amplification coefficient of thermal model of the process (hours per Celsius degree); $G^{\prime}$ - mass of the agglomerate which is produced from the layer in a working area of sintering machine (tonnes); $k^{\prime}$ - specific reaction rate (cubic meters / (hour*tonnes); $k_{0}^{\prime}$ - the pre-exponential factor; $E^{\prime}$ - current of heat needed to complete furnace-charge sintering; $c^{p}$ - mean specific heat of a solid phase in the layer (kcal / (hour*Celsius degree*tonnes); $Q_{i}, C_{i}$ - currents of heat getting off fluidized bed (also known as pseudoliquid layer) with solid and gas phases mentioned to 1 Celsius degree (kcal / (hour*Celsius degree*tonnes); $k_{T P}$ thermal losses coefficient (kcal / (hour*Celsius degree)). This model contains the macro kinetic constants (reaction rate constant, the pre-exponential factor, and activation energy $\mathrm{E}^{\prime}$ ), and thermodynamic coefficients (heat of reactions, thermal capacities, thermal expansion, and reduced heat flow). The above mentioned results in a complex mathematical apparatus (Equation 1), and a development timeline that becomes so elongated that the project may not be useful. Also, schematically the same processes may have different technological conditions and this lowers the unification ratio of solutions.

In this article, the suggested method does not focus on the definition of the observed variable dependencies on each perturbation, but on the study of the behavior of the output variable, conditionally accepting that the system is affected by some common parameter (factor) that is the resultant action of the perturbation. Suggested in article, is a method that is based on the "black box" idea, developed by Norbert Wiener and William Ashby (Wiener, 1948, Ashby, 1957).

Every physical system has an indefinitely large number of possible inputs, by which the experimenter may exert action on the Black Box (we mean Ashby's "Black box"). Equally, it has an indefinitely large number of possible outputs, for which the experimenter can use recording instruments. If the investigation is to be orderly, the set of inputs to be used and outputs to be observed must be decided upon, at least provisionally.

One can imitate any unknown nonlinear transducer by a sum of linear terms, each of fixed characteristics and with an adjustable coefficient. The "Black box" concept allows one to determine stimulus and response, and then calculate their ratio. The method is described in the following.

\section{Receiving series of a ramp}

The lines of a ramp are received by way of active or passive experimentation with various combinations of input factors that significantly influence a studied parameter. The qualitative assessment of the significance of input factors on the studied parameter can be carried out on the basis of existing technological modes, flow charts (instructions), and experience of conducting a technological process. The quantitative assessment of the significance of the input factor on the studied parameter can be defined by a known criterion, such as a series criterion or inversion criterion, while previously having set a significance value, $\alpha$.

It is necessary to note beforehand that after obtaining results an improved control performance or reduced number of control parameters can be achieved by expanding or reducing the list of significant input factors, with the significance value $\alpha$ also changing respectively. In this case, the synthesis of an adaptive digital algorithm for dynamic control of automatic control system parameters is repeated. Receiving ramp lines using the specified method will thereafter allow for a set of control parameters that provide high-quality regulation in all experimentally received ranges of incoming perturbations. The variation ranges of significant incoming perturbations are selected with some allowances, e.g., minimum values should not be less than the cumulative error of measurement and transformation channels.

As a result, a number of ramps emerge that adequately cover the range of total change from incoming perturbations and according to the suggested method, these are the "base ramps". 
For the studied variable, $y$, the temperature in the ignition zone of the camera's furnace charge for agglomeration was recorded.

\section{Building a system of mathematical models}

Developing a system of mathematical models of a ramp was carried out by approximating the basic lines of a ramp, obtain in the previous stage, with the use of the Kalman's (Kalman, 1960).

The vector differential equation of a stationary system can be readily transformed into the more convenient discrete, based on Kalman.

The steps in the methods involve the following:

1. When in service, the values of input and output parameters are recorded at strictly fixed intervals of time;

2. The type of the analytical model chosen is the simplest one, which is written in the form of the differential equation of this or that order;

3. The coefficients of a difference (equation (2)) are defined by the results of the experiment, and the accepted type of model by the minimum of the sum of squared deviations;

4. The differential equation is solved and the resulting dynamic characteristics are compared to the experiment; and

5. At significant deviations the calculation is repeated using a higher order differential equation.

Collating the dynamics, identified by the above technique, to order the performance of static identification, testified their analogy. The difference consisted only in models; the static model was described by an algebraic equation, the dynamic model, by a differential equation. For the differential linear equation of the " $\mathrm{k}$ " order, there is a differential equation analog in the form of the following:

$$
y_{n}=A_{0} y_{n-1}+A_{1} y_{n-2}+\ldots+A_{k-1} y_{n-k}+B_{0} x_{n-1}+B_{1} y_{n-2}+\ldots+B_{k-1} y_{n-k}, \text { (2) }
$$

Where $y$ - The simulated value of parameter, $x$ - experimental value of parameter, $n,(n-1),(n-2), \ldots$, $(n-k)$ - No of points of the experiment and corresponding calculations, $A, B$ are the coefficients of the difference equation.

This was accepted as the basis of the model for dynamic identification. Since the order of an identified object is initially unknown, the process of receiving a model began with the simplest model, namely, the difference equation (see equation (3)) of the first order, in the form:

$y_{n}=A_{0} y_{n-1}+B_{0} x_{n-1}$.

Where the model was insufficiently adequate, it was necessary to use the differential equation of the second order, as the following model (4):

$y_{n}=A_{0} y_{n-1}+A_{1} y_{n-2}+B_{0} x_{n-1}+B_{1} y_{n-2}$.

Further, using a technique of minimization of the sum of squared deviations, i.e. the functional of the form, resulted as follows (5):

$$
F=\min \sum_{i=1}^{m}\left(y_{E i}-y_{R i}\right)^{2} \text {, }
$$

where $y_{E i}=$ experimental values; and

$y_{R i}=$ a model output.

Following, the system of the equations will be received from (6) :

$$
\frac{\partial F}{\partial A_{0}}=0 ; \quad \frac{\partial F}{\partial A_{1}}=0 ; \quad \frac{\partial F}{\partial B_{0}}=0 ; \quad \frac{\partial F}{\partial B_{1}}=0,
$$

where it was possible to define $A_{0}, A_{1}, B_{0}$, and $B_{1}$ coefficients, which satisfy the criterion of optimality. The number of equations was the same as the number of base ramps.

\section{Evaluation of Test Data}

We considered the lines of the ramp in the change of temperature at the entrance to the agglomeration zone of the metallurgical furnace-charge, and this resulted in the course of measurements with an 
interval of $5 \mathrm{~min}$ for various initial conditions in the working range from $\Theta_{b}=250{ }^{\circ} \mathrm{C}$ to $\Theta_{e}=320{ }^{\circ} \mathrm{C}$ and from $\Theta_{b}=250{ }^{\circ} \mathrm{C}$ to $\Theta_{e}=285{ }^{\circ} \mathrm{C}$ (Uzdenbayev, 2009). For measurement, we used the L-type thermocouple, the signal from which enters the analog input module of SM331 signal check modules and these have an internal built-in function of temperature compensation, implemented by a corresponding connection scheme on the basis of S7 series of Simatic microcontrollers. The cumulative error of the measurement channel was $1.17 \%$ (the sensor measurement error is $1.0 \%$, and the analog input channel transformation error is $0.6 \%$ ). The measurement results are given in Figure 1. On Figure 1 a vertical axis is a temperature, a horizontal axis is a time.

Figure: 1 - Temperature change reduced to the lowest operational point, at the entrance to the agglomeration zone of the metallurgical furnace-charge

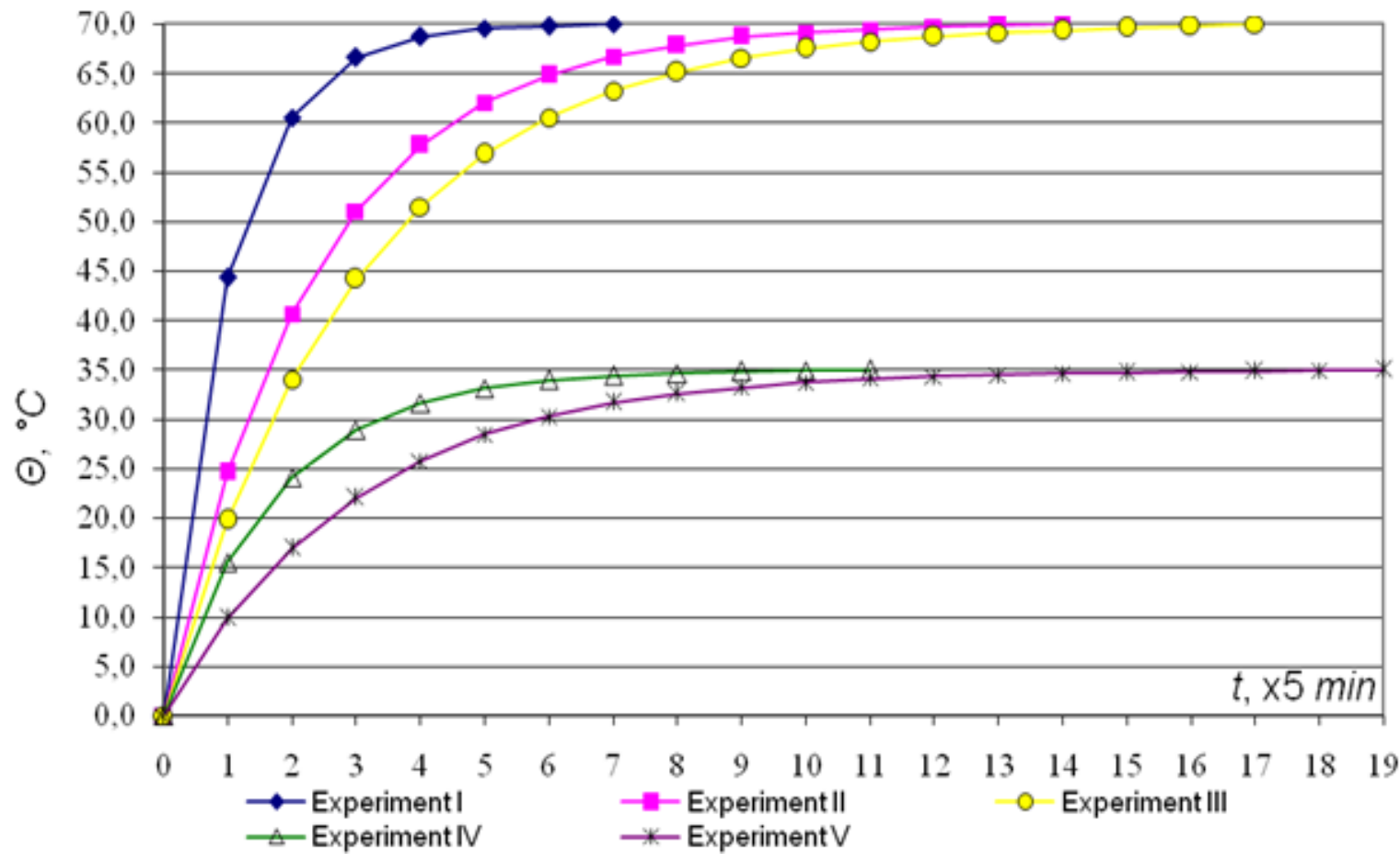

Source: Author

For identification, we used the finite-difference equation of the first order (7):

$\Theta_{n}=A_{0} \cdot \Theta_{n-1}+B_{0} \cdot \Theta_{e}$,

For minimization of the sum of squared deviations we used the function (8):

$F=\min \sum_{1}^{m}\left(\Theta_{e_{n}}-\Theta_{n}\right)^{2}$

Taking into account Equation (3) and that at the $n^{\text {th }}$ step, the model output resulting on the basis of previous steps, and $A_{0}$ and $B_{0}$ coefficients, needed to coincide with the experimental value of (9):

$F=\min \sum_{1}^{m}\left(\Theta_{n}-A_{0} \Theta_{n-1}-B_{0} \Theta_{e}\right)^{2}$ 
Thus, taking into account the data as a result of solution (8) and (9), the next system is formed by (10):

$$
\left\{\begin{array}{l}
\Theta_{n}^{1}=0,367 \cdot \Theta_{n-1}+0,633 \cdot \Theta_{e} \\
\Theta_{n}^{2}=0,649 \cdot \Theta_{n-1}+0,352 \cdot \Theta_{e} \\
\Theta_{n}^{3}=0,718 \cdot \Theta_{n-1}+0,283 \cdot \Theta_{e} \\
\Theta_{n}^{4}=0,558 \cdot \Theta_{n-1}+0,442 \cdot \Theta_{e} \\
\Theta_{n}^{5}=0,719 \cdot \Theta_{n-1}+0,281 \cdot \Theta_{e}
\end{array}\right.
$$

When checking the adequacy of models in regard to the initial data it was established that the greatest value of divergence from the results of five experiments was $0.18 \%$. Thus the cumulative error was $1.18 \%$, as a result of the existing value of $1.17 \%$, which testified to the sufficient adequacy of the model in regard to the study objective.

\section{Adaptive Control Algorithm and the Results}

An example of the traditional solution of the considered task was described as the adaptive Proportional Integral Differential (PID) controller (Takao, Yamamoto, \& Hinamoto, 2004). However, this approach needs a large set of computing operations in real-time mode and was difficult for engineering design.

Our proposed algorithm is on the Figure 2. The description of how algorithm works is given below (Uzdenbayev, 2014).

Figure 2: Algorithm of adaptable control in operational mode

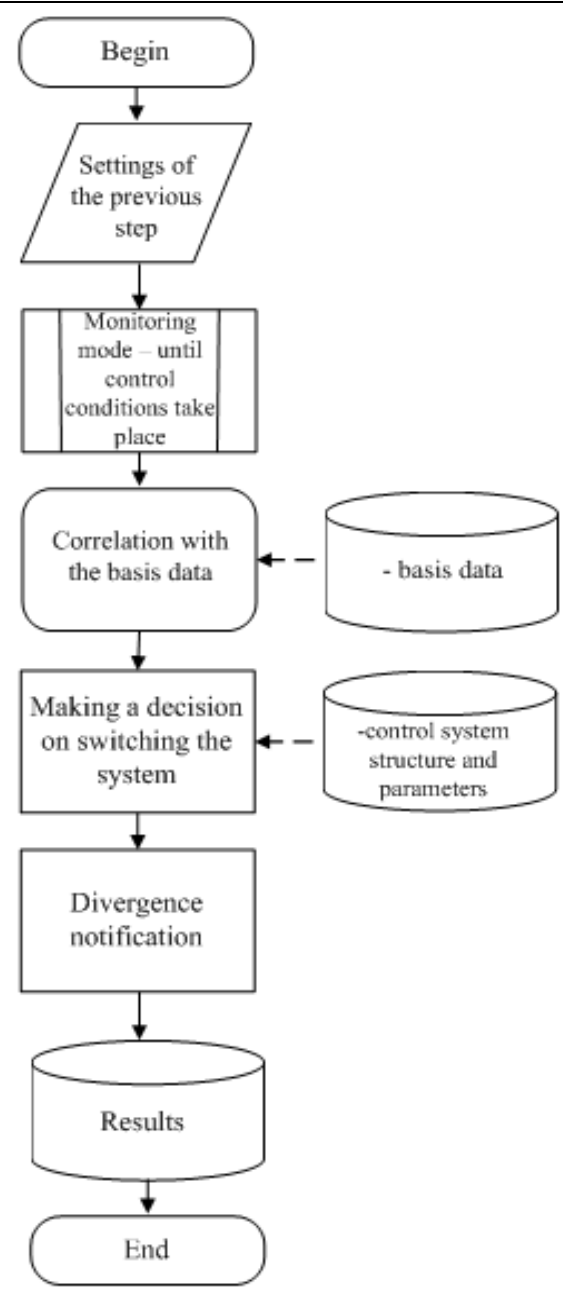

Source: Author 
Step 1. Since the initial input conditions for determining adjusted parameters at the time of switching the system into an automatic mode were not known, at the first start of the system of automatic control, it is set to the basic section with the greatest inclination of the ramp, with the corresponding parameters of regulation $(V 1)$. This section, required prompt regulation, i.e., this basic section was the most critical, because the time for regulation was small compared with other basic sections, according to the time factor. Such an approach provides the greatest transition time allowed. Conditions are described below for the extreme right of the other basis sections.

Step 2. When the value of an adjustable parameter enters the $Y_{Z N}$ dead diapason, the system functioned in the tracking mode, where the values of an adjustable parameter with pre-set discreteness were stored. For the discretization value that allows unambiguous coordinate comparability of estimated and real values of controlled parameters, we selected the discrete interval of ramp approximations for which the set accuracy of approximations was provided. For the $Y_{Z N}$ dead diapason, we selected Diapason 3 (according to the 3 sigma rule). In this mode, the system compared the actual values of the controlled parameter to the ramp lines present in the database. The actual values from the difference of the measured and recovered, with the use of a well-known control system, transferred the function values $Y_{f}=Y_{m}-Y^{\text {retr }}$ (Equation 11). This equation defined the values that would have controlled the parameter with no control input of the considered control system. Using the received values, the formula (Equation 11) determined the nearest base model.

Step 3. After the system reached the $Y_{Z V}$ level (the control zone start) for the controlled parameter, it passed into the control mode. The $Y_{Z V}$ value was established on the basis of a reaction sufficiency condition, for the considered system to provide the required quality indicators for control in the remaining time. Alternately, it was necessary to return to "organization of basis sections", as the number of considered lines of a ramp increased by one. The algorithm then worked in the abovementioned sequence.

When, in the course of operating the controller, estimates of the controlled parameter failed to enter the area of the ramp, they were recorded in a separate base, and the technologist operator was informed. Where this event was connected to input factors that were not considered during the design phase, the area of the ramp and the calculations of settings were reconsidered; and where necessary, corresponding changes to the algorithm were made.

Figure 3 shows the graphs of the transient processes that explain the operation of the algorithm. On Figure 3, the vertical axis is temperature and the horizontal axis is time. The number of basis curves equaled five, and are numbered left to right, i.e., to the decrease of inclination. At the beginning of operation of the control system, the parameters established in the previous control period, i.e., the control parameters, were configured for Basis Curve 5, and the actual curve corresponded to Basis Curve $1\left(\left|Y_{\mathrm{ZN}}\right|=3.9^{\circ} \mathrm{C}\right.$, with corresponding $Y_{\mathrm{ZV}}$ of 90 seconds).

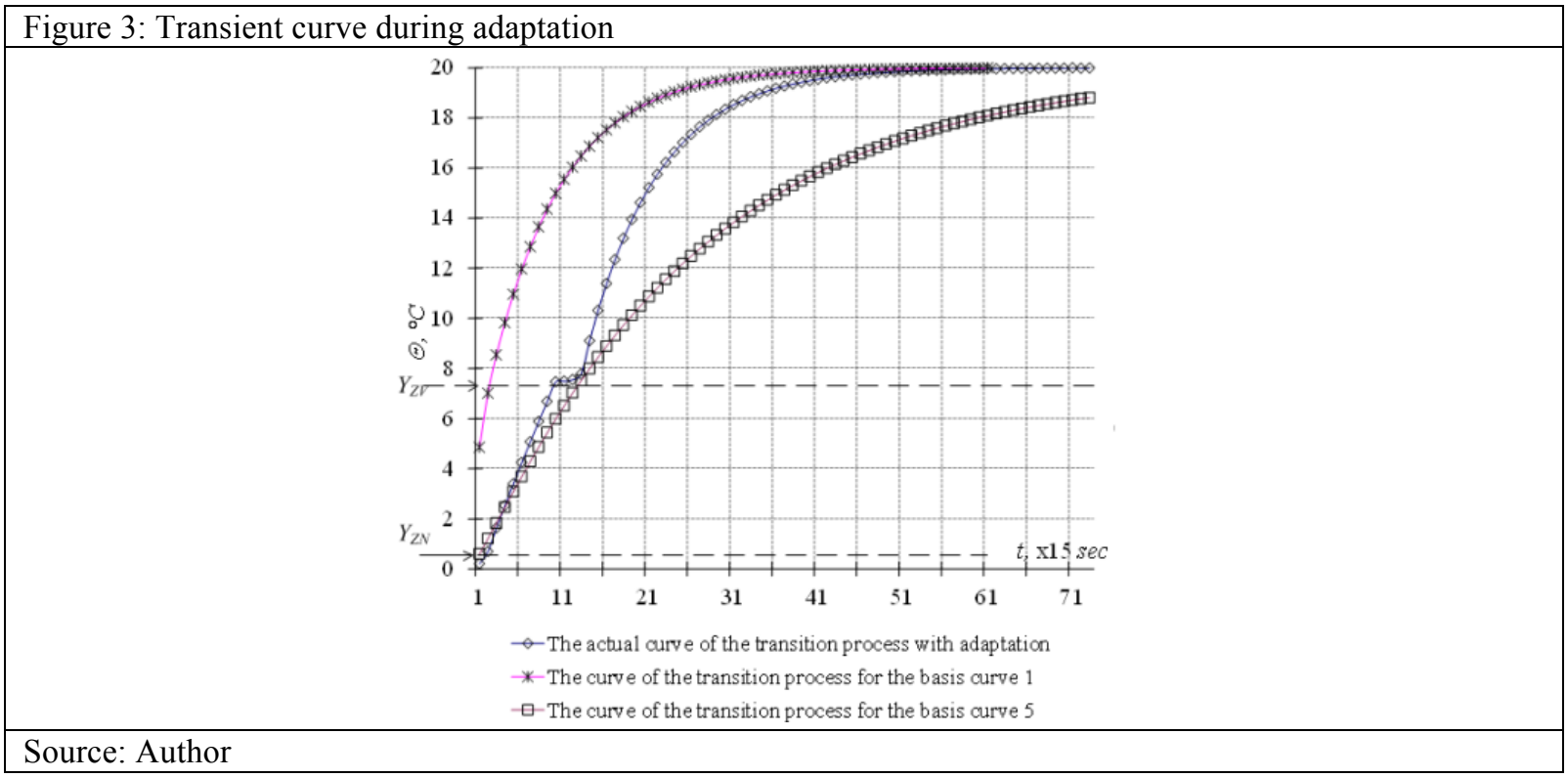




\section{Conclusion}

Developing a system of mathematical models using this method provides concrete metallurgical technology that achieves a difference of no more than $1.18 \%$ between calculated and experimental data. The method reduces the design period of an adaptive automatic regulator from five to six times (including all calculations), and also reduces control lag for the technological process from between 80 and 90 minutes to between 10 and 15 minutes.

\section{References}

Ashby, W. (1957). An Introduction to Cybernetics. London, Chapman and Hall, 295.

Burovoi, I. A., \& Eliashberg, I M. (1964). Identifikaciya himiko - metallurgicheskih proizvodstv. [Identification of the chemical and metallurgical industries]. Collection of scientific works Gintsvetmet, №21, Moskow, Metallurgiia, 5-8.

Kalman, R. E. (1960). A New Approach to Linear Filtering and Prediction Problems Trans. ASME (American Society of Mechanical Engineers), J. Basic Eng., vol. 82 D.4.

Takao, K., Yamamoto, T., \& Hinamoto, T. (2004) Design of a memory-based self-timing PID controller. 43rd IEEE Conference on Decision and Control (CDC 2004), Vol. 2, 1598-1603.

Uzdenbayev, Zh. (2009). Postroenie sistemy matematicheskih modelej s ispolzovaniem metoda dinamicheskoj identifikaciiyu. [Building a system of mathematical models using dynamic identification method]. Máteriály v Mezinárodni Vědecko-praktická konference "Vědecký pokrok na rozmezi millennium- 2009". Praha, 60-67 [Material in the international scientific - practical conference "Scientific progress on the range of millennium- 2009". Prague, 60-67].

Uzdenbayev, Zh. Sh. (2014). Adaptive Process Control Systems. LAP LAMBERT Academic Publishing is a trademark of: OmniScriptum GmbH \& Co. Saarbrucken, Germany, 105.

Wiener, N. (1948). Cybernetics: or the Control and Communication in the Animal and the Machine. Cambridge: The Technology, 194. 